\title{
Cognitive Preferences and Level of Patient Management among Physical Therapists in Northern Mindanao
}

\author{
DIONESIO A. PONGO, JR. \\ ORCID No. 0000-0002-4495-7176 \\ ldcupongo@yahoo.com.ph \\ Liceo de Cagayan University \\ Cagayan de Oro City, Philippines
}

\begin{abstract}
The call for independent practice in the Philippines for physical therapy professionals requires a satisfactory degree of cognitive preparedness needed for the clinical decision-making process and adaptation to new trends of patient management. This study aimed to determine the significant relationship between cognitive preference and the level of patient management of physical therapists in Northern Mindanao. The study makes use of descriptive correlational design. The respondents of the study were thirty (30) licensed physical therapists in Region 10 working as staff among PT facilities and those who are into private practice. The data show that clinical reasoning, screening, examination, evaluation, plan of care, procedural and educational intervention, documentation and outcome assessment has no significant relationship towards preceptive, receptive, intuitive, and systematic cognitive style of physical therapists. This is because the p-value did not reach the level of significance. However, the only diagnosis shows significant result towards preceptive, systematic, and intuitive cognitive preference. The results of the study suggest that preceptive, receptive, systematic and intuitive cognitive preference did not significantly relate to the clinical evaluation performance of physical therapists. Conversely, the ability to operate in the preceptive, systematic and intuitive mode is associated with better performance in diagnosis of patient management.
\end{abstract}

Keywords: Independent practice, clinical decision-making, patient management, cognitive preferences, physical therapists 


\section{INTRODUCTION}

In the 21 st century, the physical therapy profession worldwide has continued to grow substantially. The promotion of healthy lifestyle in the community by physical therapists becomes more relevant. In response to this transformation, the physical therapy profession in the Philippines is moving towards full autonomous practice and direct access to patient care. This development makes a physical therapist become a primary care contact, and that patients may directly seek the services of a physical therapist without a referral from a physician or other allied medical professionals. However, this call for independent practice requires a satisfactory degree of cognitive preparedness needed for the clinical decision-making process and adaptation to new trends of patient management.

Cognitive style or preference is defined as an individual's preferred way of thinking and organizing information. Identification of cognitive preferences and level of clinical practice regarding patient management of physical therapists is imperative because of their involvement in primary care contact.

Patient management is a vital key in the practice of physical therapy profession in pursuance of obtaining a concrete patient intervention. It involves a spectrum of processes involving dynamic and complex clinical interpretation and analytical thinking that involves making decision and determinations in the perspective of patient management (Kisner, 2013). The components of patient management model include examination, evaluation, diagnosis, prognosis and plan of care and interventions. This process helps a physical therapist organize, prioritize data and plan effective treatments well-suited with the problems and goals of the patient/ client. Because of the high level of clinical thinking and reasoning required by a physical therapist during patient management, the researcher believes that the preferred cognitive technique of physical therapists may have some repercussion towards patient management conduct and clinical judgment.

In Northern Mindanao, physical therapy practice remains traditional. The physiatrist conducts an examination, evaluation, and determines diagnosis, prognosis, and plan of care and formulates interventions. The physiatrist then assigns the physical therapist to perform therapeutic interventions to the patient/client. Moreover, the majority of the treatment procedures performed by the physical therapists are planned by a physiatrist based on the medical diagnosis of the patient's problem. A physical therapist rarely performs the process of examination, evaluation, determination of diagnosis, prognosis and plan of care and lastly treatment interventions. Autonomy is seldom on the part of the physical therapist to perform the process of clinical decision-making. 
Identification of standards of practice of physical therapist on clinical decisionmaking on patient management will help us evaluate their clinical competency that is important in maintaining safe, high standards and cost-effective health care. Furthermore, this will also delineate the present scope of physical therapy practice, and provide data that will direct the curricula of physical therapy professionals, the post-baccalaureate as well as continuing education programs.

The primary purpose of the researcher in conducting this study is to determine the significant relationship between the cognitive preferences and the level of patient management among physical therapists in Northern Mindanao.

\section{CONCEPTUAL FRAMEWORK}

This study is based on the concept of Cognitive Preference developed by McKenny and Keen (1974) as a way of thinking and synthesizing information as a foundation for physical therapy clinical decision making during patient management. This study is also based on the Comprehensive Outcomes-Oriented Model of Patient Management (Figure 1.) developed by the American Physical Therapy Association indicated in their Guide to Physical Therapist Practice by Kisner (2013) as a foundation for organizing and prioritizing patient's information and planning effective treatment during the patient encounter by a physical therapist.

McKenney and Keen's concept reflected four styles of cognition, namely preceptive, receptive, systematic and intuitive cognitive style. Two of these are related to the data gathering phase and two to the information-processing phase of decision making.

\section{Data Gathering Phase}

According to (McKenny, 1974) the data gathering phase includes receptive and preceptive cognitive style. Receptive individuals are more responsive to the impulse itself. The data are analyzed individually and collectively before a final determination is made of how to organized and use them (O'Sullivan, 2014). Judgment or decision is suspended until all possible data have been collected, paying attention to detail, and attending to the implications of each piece of data individually (APTA, 2001).

While "preceptive thinkers will seek and respond to ongoing cues and patterns, defining and organizing problems early. They focus on connections between things. Their precepts act as cues for both gathering and categorizing the data they find. (Martens, 1975) . Information Processing Phase.

Individuals differ not only in their methods of gathering data but also in their sequence of the data analysis. The information processing phase, which relates 
to problem-solving reflects these differences ( McKenney \& Keen 1974). By this difference, individuals range from systematic to intuitive thinkers. The systematic individuals follow consciously a procedural approach, defining problems and constraints early, performing an ordered search for information, and finishing one step at a time before moving to the next. While "intuitive thinkers, employ a strategy which is more of solution testing and trial-and-error. Intuitive thinkers rely on pattern recognition and will point to solutions that have worked well with the currently perceived pattern (Matzler, 2007).

Clinical decision-making during patient management in physical therapy involves 2 phases, namely: the data gathering phase and the information-processing phase. Data gathering takes place during the examination, the first part of the patient management process. The patient/client history of the problem is revealed during the examination. The patient also answers the question relating to the system of the body. On the later part of the examination, to provide objective data, the therapist then would decide what particular test and measures to administer to determine accurately the degree of specific function and dysfunction present.

After examination, evaluation follows during which physical therapists conduct an assessment of all the information collected from the patient history, the review of systems and physical therapy tests. This information will then be analyzed to determine the patient's problem as a basis for formulating treatment interventions.

An individual's style may be either predominantly preceptive or receptive in the process of gathering information and either predominately systematic or intuitive in evaluating that information. Thus, an individual may show a preference for one of four possible styles; intuitive-preceptive, intuitive-receptive, systematic-preceptive, or systematic- receptive.( Martens, 1975).

Another model that supports this study is on the Comprehensive OutcomesOriented Model of Patient Management (Figure 1.) developed by the American Physical Therapy Association indicated in their Guide to Physical Therapist Practice by Kisner (2013) as a foundation for organizing and prioritizing patient's information and planning effective treatment during the patient encounter by a physical therapist. The patient management model is designed to guide a practitioner through a systematic series of steps and clinical decision-making for the purpose of helping a patient achieve the highest level of function possible. The overall goal of the patient management process is the achievement of a realistic functional result for the patient.

The process of patient management has five basic components (APTA, 2001). These consist of 1) A comprehensive examination, 2) Evaluation of data gathered, 3) Formulation of a physical therapy diagnosis 4) generation of a prognosis and plan of care and 5) Carrying out physical therapy interventions. Physical therapists that 
follow this process will be able to determine whether the patient or clients require the service of physical therapists. The process of patient management will also help the physical therapists to formulate a plan of care in cooperation of the patient/client or other members involved (APTA, 2011).

\section{Examination}

The first component of the patient management model is a comprehensive examination of the patient. The examination consists of three components (APTA, 2001): 1) the patient's history, 2) review of the system, and 3) physical therapy tests and measures. The examination is a process by which a physical therapist acquires information about a patient's reason for coming to the rehabilitation center. The examination process involves both comprehensive screening and specific diagnostic testing. The physical therapist collects enough data about the patient's potential problems ultimately to formulate a diagnosis and determine whether physical therapy interventions can treat these problems.

\section{Evaluation}

The second component of the patient management model is the evaluation. Evaluation is a process characterized by the interpretation of collected data. The process involves the classification and synthesizing of information as a basis for developing physical therapy diagnosis (APTA, 2001).

\section{Diagnosis}

Diagnosis is the third element of the patient management process. The diagnosis provides a basis for formulating patient's plan of care, interventions and prognosis (APTA, 2001). Data collected from the examination process will be evaluated to determine the probable cause of patient/client problems. The same data collected will serve as the basis for developing a prognosis and choosing physical therapy interventions strategies during the development of the plan of care. (O'Sullivan, 2014).

\section{Prognosis and Plan of Care}

The fourth component is prognosis and plan of care. A prognosis is a projection of patient's best possible level of function as a consequence of physical therapy interventions. It also includes the estimation of time to reach the goal (APTA, 2001).

The plan of care is an integral component of the prognosis. It delineates the following: 1) Anticipated goals 2) Expected functional outcomes that are meaningful, utilitarian, sustainable, and measurable, 3) Extent of improvement predicted and 
length of time necessary to reach that level, 4) Specific interventions, 5) projected frequency and duration of physical therapy interventions, and 5) detailed discharge plans (APTA, 2001).

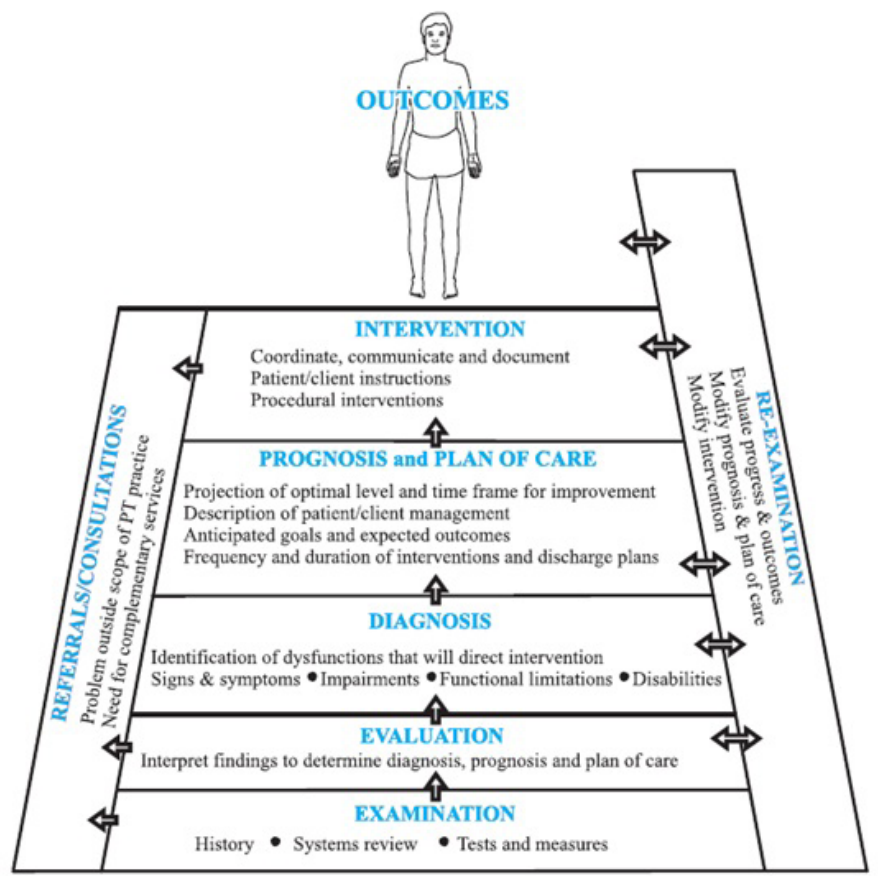

Figure 1. Comprehensive Outcomes-Oriented Model of Patient Management (Kisner and Colby, Therapeutic Exercises: Foundations and Techniques, 2013)

\section{Intervention}

Intervention is the 5 th component of patient management. Intervention refers to any purposeful interaction of the patient/client with the physical therapist and other persons concerned in the overall management of the patient/client, using various physical therapy (APTA, 2001). There are three broad areas of intervention that occur during patient management (APTA, 2001): 1) Coordination, communication, and documentation 2) Procedural interventions and 3) Patient-related instruction. Each of these areas is an essential aspect of the intervention phase of patient management. A therapist's skillful, creative use of all three components of the intervention, coupled with vigilant re-examination and re-evaluation of the effectiveness of the interventions selected, paves the way for successful outcomes and a patient's discharge from physical therapy services. 


\section{Outcomes}

Outcomes determine the efficacy of treatment. Simply stated, outcomes are results. The quality, efficacy, and cost-effectiveness of physical therapy services is assessed through measurement of outcomes. Throughout an episode of physical therapy care, that is, intermittently during treatment and at the conclusion of treatment outcomes are monitored (Ozer, 2000).

INDEPENDENT VARIABLES

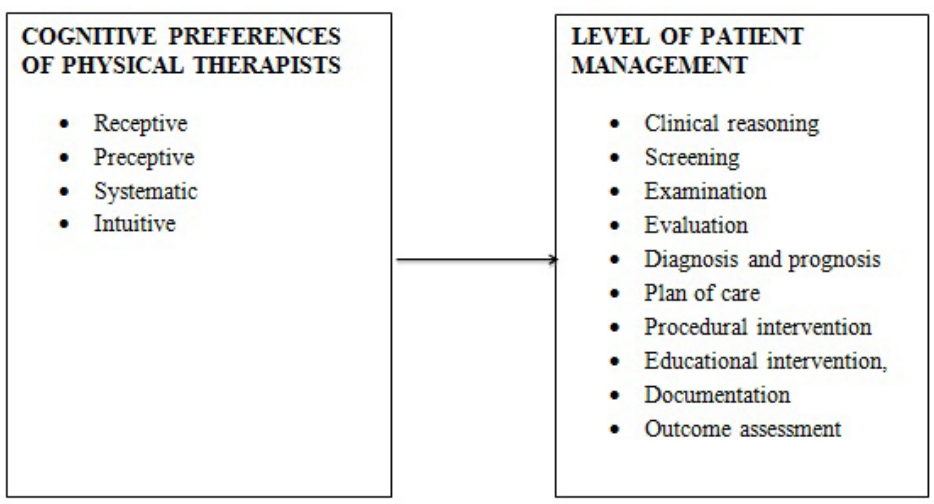

Figure2. Schematic Presentation Showing the Interplay of Variables in the Statement of the Problem of this Study

\section{OBJECTIVES OF THE STUDY}

This study seeks to determine the relationship between the cognitive preferences and the level of patient management among physical therapists in Northern Mindanao. Specifically, this study aims to: 1) determine the cognitive preferences for physical therapists in terms of perceptive, receptive, systematic and intuitive preferences; 2 ) assess the level of patient management of the physical therapists in terms of clinical reasoning, screening, examination, evaluation, diagnosis and prognosis, plan of care, procedural intervention, educational intervention, documentation, and outcome assessment; and 3) relate cognitive preferences and the level of patient management of physical therapists. 


\section{METHODOLOGY}

\section{Research Setting}

The study was conducted within Northern Mindanao (Region 10) and involve licensed physical therapists working as staff among PT facilities and those who are into private practice.

\section{Research Design}

This research utilized descriptive-correlational design. A correlational study examines the extent to which differences in one characteristics or variables related to differences in one or more other characteristics or variables (Leedy \& Ormrod, 2005). In this particular study, cognitive preferences and the level of patient management were the variables that need to determine whether or not such relationship exists.

\section{Respondents and Sampling Procedure}

The respondents of the study were licensed physical therapists working as fulltime staff among physical therapy facilities and physical therapists who are into private practice in Northern Mindanao. The study employed convenience sampling in determining the respondents of the study.

\section{Research Instrumentation}

This study adapted the research instrument of Bella J. May and Jancis K. Dennis (1991) on Expert Decision Making in Physical Therapy-A Survey of Practitioners to determine the cognitive preferences of the physical therapist. The study also used the Physical Therapist Clinical Performance Instrument of the American Physical Therapy Association (APTA), Department of Physical Therapy Education, 2006 with the exclusion of some indicators such as financial resources and direction and supervision of personnel that are not applicable in the physical therapy practice in the Philippine setting.

A forty-eight (48) item survey is placed in Part I to determine the cognitive preferences of the physical therapist in clinical decision-making. Part II of the research instrument focused on the level of practice in the clinical decision-making process on patient management of physical therapists. Indicators for the patient management used sample behaviors on clinical reasoning, screening, examination, evaluation, diagnosis, and prognosis, plan of care, procedural intervention, educational intervention, documentation, and outcomes assessment. 


\section{Reliability and Validity}

Part II of the research instrument statistically analyzed for reliability test and revealed a Cronbach's Alpha score of 0.962 , interpreted as reliable.

\section{Data Gathering Procedure}

The researcher sent a letter to the Dean of Graduate Studies for approval to conduct the study. The researcher then sent another letter to the Head of the physical therapy facility asking permission to conduct the study. Upon approval, the researcher approached the respondents. Before the actual interview and observation process, the researcher explained the purposes and procedures to be undertaken by the respondents and obtained their consent by letting them sign the consent form.

The researcher was directly responsible for the administration of the questionnaires on the cognitive preferences of the respondents. The respondents directly rate the 48- item statements in the questionnaire to assess their cognitive preferences in the clinical decision making. Also to determine the level of practice in clinical decisionmaking process on patient management, the researcher conducted direct observations to evaluate the level of practice on patient management regarding clinical reasoning, screening, examination, diagnosis, and prognosis, plan of care, procedural and educational interventions, documentation and outcome assessment.

\section{Statistical Techniques}

Computation of weighted means was done to determine the cognitive preferences and level of patient management of the physical therapists. Pearson correlation coefficient was done to determine the relationship between the cognitive preferences and the level of patient management of physical therapists.

\section{Scoring Procedure}

The following scoring system used with their corresponding responses for PART I of the questionnaire, as to determine the cognitive preferences of the physical therapist in clinical decision-making.

3.5-4.0 - if you relate strongly to the statement, you will strongly agree with it.

2.5-3.49 - if you relate to the statement, but it is not highly characteristics of you, you will agree with it

1.5- 2.49 - if you do not relate to the statement, but you know you do this occasionally, you will disagree with it.

1.0-1.49 - if you do not relate to the statement at all, you will strongly disagree with it. 
The following scoring system used with their corresponding responses for PART II of the questionnaire, as to determine the level of practice in the clinical decisionmaking process on patient management.

$3.5-4.0$ Always As a physical therapist, you practice clinical decision making process at all times during patient management.

2.5-3.49 Mostly As a physical therapist, you practice clinical decision making process largely of the times during patient management.

1.5-2.49 Occasionaly As a physical therapist, you practice clinical decision making process irregularly of the time during patient encounter.

1.0-1.49 Seldom As a physical therapist, you practice clinical decision making process rarely of the time during patient management.

\section{Categorization of Variables}

The interview statements of part I of the questionnaire was screen and identify that match the cognitive style descriptions.
A. Preceptive cognitive preference related instrument items are no $1,17,24,31,35,41$ and 47 ,
B. receptive cognitive preference related instrument items are no $5,7,10,11,27,29$, and 44,
C. systematic cognitive preference related instrument items are no $13,16,30,37,40$, and 43 , and
D. intuitive cognitive preference related instrument items are no 2,4,22, and 23 respectively.

Individual scores were computed by averaging the responses to items within each category (preceptive, receptive, systematic, and intuitive).

\section{RESULTS AND DISCUSSION}

Table 1 presents the cognitive preference of physical therapists. The overall mean score of receptive cognitive preference is slightly higher than preceptive cognitive preference. The data suggest that physical therapists in the region are more likely to practice receptive thinking during information gathering phase of examination as such they focus more on detail than relationship and derive information from direct examination than fitting to their precepts (Mckenny and Keen, 1974).

Throughout the assessment, receptive thinkers keep a mental checklist to be sure they are doing everything they need to do and use physical assessment to check out initial ideas about the patient's problem. 
Table 1. Cognitive Preferences of Physical Therapists

\begin{tabular}{lccc}
\hline & Cognitive Preference & Mean & Verbal Description \\
\hline Preceptive & 3.13 & Agree \\
Receptive & 3.19 & Agree \\
Systematic & 3.11 & Agree \\
Intuitive & 2.66 & Agree \\
\hline
\end{tabular}

Once they have a picture of the patient's problems, they stop collecting information to confirm its findings.

The receptive cognitive style also has its disadvantage. The receptive thinker may fail to shape detail into a coherent whole. A bunch of information does not guarantee to find the solution to the problem that is if the examiner fails to recognize its relevance. It is suggested that physical therapists should carefully assess patients individually gather relevant data that will help solve the problem and determine the course of action.

In contrast, preceptive thinkers gather information differently from that of receptive thinkers. When they get a referral, they get a mental image of the patient. They start to make judgment about the patient's problem as soon as they see the patient without going into minuscule details in the assessment. Moreover, when developing problem list, they tend to focus on a few pieces of information that they consider critical.

Likewise, preceptive thinkers have its disadvantage as well. Its drawback includes the risk of overlooking the potential meaning of the data thus preceptive individual easily ignores relevant detail. Dennis and Bella (1991) said that students who operated primarily in the preceptive mode were less likely to determine accurately a simulated patient's problems.

To determine the patient's problem accurately, it is suggested that physical therapists should carefully assess patient individually and follow the process of clinical decision making to avoid errors during patient management

The overall mean score of systematic is higher than intuitive cognitive preference. This only mean to say that physical therapists plan their assessment in a systematic manner and complete evaluation before making clinical decisions about the treatment. An organized decision-making process based on a systematic approach helps the physical therapists deals with difficult problems and would likely produce a better result (http://www.academia.edu/6656509/A_Systematic_Approach_to_ Decision_Making).

Therefore, it is commonly used method by practicing physical therapists in the region when processing patient information. While decision-making based on intuition is not commonly practiced, intuitive decision-making is inaccurate, unreliable and insufficient to use (Matzler, 2007). 
Table 2. Level Of Patient Management Of Physical Therapists

\begin{tabular}{lll}
\hline \multicolumn{1}{c}{ Level of Patient Management } & Mean & Verbal Description \\
\hline Clinical reasoning & & \\
Screening & 3.53 & Always \\
Examination & 3.54 & Always \\
Evaluation & 3.54 & Always \\
Diagnosis and prognosis & 3.48 & Mostly \\
Plan of care & 3.33 & Mostly \\
Procedural intervention & 3.54 & Always \\
Educationalintervention & 3.71 & Always \\
Documentation & 3.67 & Always \\
Outcome assessment & 3.40 & Mostly \\
\hline
\end{tabular}

Table 2 presents the level of patient management of physical therapists. It is important to note that physical therapists "always" demonstrate clinical reasoning, screening, examination, plan of care, procedural interventions, educational interventions, and outcome assessment during patient management. However, evaluation, diagnosis and prognosis and documentation is "mostly" done by physical therapists. The data denote that the practice of evaluation, diagnosis and prognosis and documentation on patient management needs improvement.

Though physical therapists in the region "always" perform the patient examination, their ability to synthesize the data from the history, system review, and tests and measures and make clinical judgments based on the data from examination needs improvement. They perform poorly on this aspect for the reason they seldom practice this element of patient management. There is nothing left to evaluate since the patient is already diagnosed by the physiatrist. Because of this, physical therapists ability to interpret collected data and make clinical decisions is poorly developed.

In the same manner, physical therapists in the region should improve their level of patient management on diagnosis. They perform poorly on this element of patient management because they seldom conduct the evaluation that is a prerequisite in establishing the patient diagnosis. The diagnostic process is a complex sequence of actions and decisions that begins with the collection of data, analysis and interpretation of all relevant data collected and formation of a diagnostic hypothesis (Kisner, 2013). In other words, to be able to diagnose the patient's problem, physical therapists should evaluate all pertinent information, including data from the patient history, system review and tests and measures.Establishing the patient diagnosis is important because it guides the physical therapists in determining therapeutic interventions (Goodman, 2009).

For them to develop their critical thinking ability, physical therapists in the region should perform the evaluation in all patient encounter by synthesizing all the results of examination data to diagnose the patient/client problem as well as to determine 
the prognosis and treatment plan.

Likewise, their level of patient management on documentation should improve. There is no known regulatory agencies and third-party payers that require or review patient documents. Therefore, justification of physical therapy services through accurate documentation is not emphasized on the physical therapy practice in the region.

Table 3 presents the correlation between cognitive preferences and the level of patient management among physical therapists. The data show that clinical reasoning, screening, examination, evaluation, plan of care, procedural and educational intervention, documentation and outcome assessment has no significant relationship towards the preceptive cognitive style of physical therapists. This is because the $\mathrm{p}$-value did not reach the level of significance ( $\mathrm{p}$-value $=0.05$ ).

Table 3. Correlation between Cognitive Preferences and the Level of Patient Management among Physical Therapists

\begin{tabular}{|c|c|c|c|c|}
\hline Cognitive Preference & Patient Management & $\begin{array}{c}\text { Pearson } \\
\text { Correlation }\end{array}$ & P-value & Interpretation \\
\hline \multirow{10}{*}{ Preceptive } & Clinical reasoning & 0.132 & 0.486 & Not Significant \\
\hline & Screening & -0.172 & 0.365 & Not Significant \\
\hline & Examination & -0.149 & 0.433 & Not Significant \\
\hline & Evaluation & -0.140 & 0.459 & Not Significant \\
\hline & Diagnosis and prognosis & 0.377 & 0.040 & Significant \\
\hline & Plan of care & -0.128 & 0.499 & Not Significant \\
\hline & Procedural intervention & -0.137 & 0.470 & Not Significant \\
\hline & Educational intervention & -0.131 & 0.489 & Not Significant \\
\hline & Documentation & -0.233 & 0.216 & Not Significant \\
\hline & Outcome assessment & -0.111 & 0.558 & Not Significant \\
\hline \multirow{10}{*}{ Receptive } & Climical reasoning & 0.034 & 0.859 & Not Significant \\
\hline & Screening & -0.291 & 0.118 & Not Significant \\
\hline & Examination & -0.199 & 0.292 & Not Significant \\
\hline & Evaluation & -0.266 & 0.156 & Not Significant \\
\hline & Diagnosis and prognosis & 0.303 & 0.104 & Not Significant \\
\hline & Plan of care & -0.284 & 0.128 & Not Significant \\
\hline & Procedural intervention & -0.252 & 0.179 & Not Significant \\
\hline & Educational intervention & -0.262 & 0.161 & Not Significant \\
\hline & Documentation & -0.295 & 0.113 & Not Significant \\
\hline & Outcome assessment & -0.190 & 0.314 & Not Significant \\
\hline \multirow{10}{*}{ Systematic } & Clinical reasoning & 0.118 & 0.534 & Not Significant \\
\hline & Screening & -0.127 & 0.503 & Not Significant \\
\hline & Examination & -0.205 & 0.278 & Not Significant \\
\hline & Evaluation & -0.190 & 0.314 & Not Significant \\
\hline & Diagnosis and prognosis & 0.387 & 0.035 & Significant \\
\hline & Plan of care & -0.211 & 0.263 & Not Significant \\
\hline & Procedural intervention & -0.145 & 0.444 & Not Significant \\
\hline & Educational intervention & -0.242 & 0.197 & Not Significant \\
\hline & Documentation & -0.199 & 0.291 & Not Significant \\
\hline & Outcome assessment & -0.237 & 0.207 & Not Significant \\
\hline \multirow{10}{*}{ Intuitive } & Clinical reasoning & 0.162 & 0.394 & Not Significant \\
\hline & Screening & -0.146 & 0.442 & Not Significant \\
\hline & Examination & -0.072 & 0.706 & Not Significant \\
\hline & Evaluation & -0.079 & 0.679 & Not Significant \\
\hline & Diagnosis and prognosis & 0.430 & 0.018 & Significant \\
\hline & Plan of care & -0.144 & 0.449 & Not Significant \\
\hline & Procedural intervention & -0.162 & 0.392 & Not Significant \\
\hline & Educational intervention & -0.167 & 0.379 & Not Significant \\
\hline & Documentation & -0.182 & 0.335 & Not Significant \\
\hline & Outcome assessment & -0.003 & 0.986 & Not Significant \\
\hline
\end{tabular}


However, only diagnosis and prognosis showed significant result ( $\mathrm{p}$-value $=$ 0.040) towards the preceptive cognitive preference of physical therapists. Therefore, physical therapist who operates under preceptive cognitive preference performs better in diagnostic part of patient management. Preceptive thinkers tend to look for cues in a data set and focuse on the relationship. This ability helps them perform better in integrating and evaluating the data obtained from the examination. Utilizing their forward reasoning approach, years of experience and knowledge of the disease and injury, mechanism of injury and signs and symptoms, preceptive physical therapists can diagnose the patient problem more easily. Also, preceptive thinkers can easily recognize pattern faster and more efficient and thus able to diagnose the patient problem without difficulty (Edwards et al. 2004).

Dennis and May (1991) mentioned that students who operated primarily in the preceptive mode were less likely to determine a simulated patient's problem accurately. This is true because preceptive thinkers rely on much of experience in gathering and categorizing information and students does not have the skill yet to use it.

The table also shows no significant relationship present between receptive cognitive preference and the level of patient management among physical therapists.

In the same way, clinical reasoning, screening, examination, evaluation, plan of care, procedural and educational intervention, documentation and outcome assessment shows no significant relationship to systematic and intuitive cognitive style of physical therapists. However, only diagnosis demonstrate significant result towards systematic $(\mathrm{p}$-value $=0.035)$ and intuitive $(\mathrm{p}$-value $=0.018)$ cognitive style of physical therapists. Therefore, physical therapist that operates under systematic and intuitive cognitive style performs better in the diagnostic part of patient management. The data also imply that physical therapists utilize both cognitive styles in processing information that would lead them to make a diagnosis. Systematic physical therapists can plan and follow a systematic process and able to complete patient evaluation before making clinical decisions. On the other hand, intuitive physical therapists rely much on unverbalized cues and hunches, defining the solution regarding fit. When confronted with a problem physical therapists make use of a style that would fit the situation at hand (Mckenny and Keen, 1974). Their cognitive preference, therefore, depends on the task. In this particular case the formulation of patient's diagnosis.

The data also show that preceptive, receptive, systematic and intuitive cognitive preference of physical therapists has no significant effect on the level of practice on clinical reasoning on patient management. It should be borne in mind that patient management is a vital key in the physical therapy practice to obtain concrete patient interventions. Patient management involves a spectrum of processes involving dynamic and complex clinical reasoning and analytical thinking that involves making judgments and determinations in the context of patient care (Kisner, 2013). The 
ability of the physical therapist to conduct a comprehensive examination, evaluation of data collected, determination of diagnosis and prognosis, the establishment of a plan of care and formulation and implementation of appropriate interventions are highly cognitive in the process that involved clinical thinking and reasoning skills required by a physical therapist during patient management. It is a sad thing to know that respondent's cognitive style has no influence on their level of practice in clinical reasoning on patient management. This means that physical therapists ability to conduct examination, evaluation, determination of diagnosis and prognosis, development of a plan of care and selection, implementation, and modification of therapeutic exercise interventions, ability to make a timely decision and judgment on practice is questionable. Clinical reasoning is relevant because the physical therapist has to make a wide variety of decision in his/her daily clinical practice (Edwards et al. 2004). Their ability to make timely decision and judgment is important in attaining meaningful, functional outcomes by the patient. Aside from that clinical reasoning is important because independent and responsible decision making is now regarded as one characteristic of physical therapists needed to practice their profession autonomously.

\section{CONCLUSIONS}

Based of the findings, the following conclusions were highlighted: 1) preceptive/ systematic cognitive preference is used commonly by physical therapists during information gathering and information processing phase; 2) the indicators for patient management of physical therapists are "always" demonstrated based on the standard of physical therapy clinical practice, except evaluation, diagnosis and prognosis and documentation that is" mostly demonstrated; and 3) preceptive, receptive, systematic and intuitive cognitive preference did not significantly relate to the clinical evaluation performance of physical therapists. The ability to operate in the preceptive, receptive, systematic and intuitive mode is not associated with better performance in clinical reasoning, screening, examination, evaluation, plan of care, procedural and educational interventions, documentation, and outcome assessment of patient management. However, the ability to operate in the preceptive, systematic and intuitive manner is associated with better performance in formulating patient's diagnosis. 


\section{LITERATURE CITED}

American Physical Therapy Association

2011 Today's Physical Therapist: A Comprehensive Review of a 21st

Century Health Care Profession (www. moveforward pt.com).

American Physical Therapy Association

2001 Guide to Physical Therapist Practice. (2nd Ed.). Phys Ther, 81:9-744.

Edwards, I. \& Jones, M.

2004 Clinical Reasoning Strategies in Physical Therapy. Journal of Physical Therapy.

Goodman, C. \& Snyder, T.

2009 Differential Diagnosis for Physical Therapists: Screening for Referral. (4th Ed.). Elsevier (Singapore) Pte Ltd.

http://www.academia.edu/6656509/A_Systematic_Approach_to_Decision_Making Kisner, C. \& Colby L.

2013 Therapeutic Exercise: Foundations and Techniques. (6th Ed.). F.A. Davis Company, 1915 Arch Street, Philadelphia.

Leedy, P.D. \& Ormrod, J.E.

2005 Practical Research. Planning and Design. (8th Ed.). Pearson Prentice Hall, New Jersey.

Leighton, R.D., Sheldon, M.R.

1997 Model for teaching clinical decision making in a physical therapy professional curriculum. J Physical Therapy Education, 11(Fall):23.

Martens, K.

1975 Cognitive Style: Recognizing Individual Differences.

Matzler, K., Ballom, F. \& Mooradlan, T.

2007 Intuitive Decision Making. MIT Sloan Management Review. Retrieved April 6, 2010, from http://sloanreview.mit.edu/the-magazine/files/ pdfs/49108SxW.pdf 
Asian Journal of Health Volume 5

May, B.J. \& Dennis, J.K.

1991 Expert Decision Making in Physical Therapy-A survey of Practitioners. Phys Ther. 71:190-202.

McKenney, J.L. \& Keen, P.G.W.

1974 Managerial Style.

O'Sullivan S.B., Schmitz, T.J.

2014 Physical Rehabilitation: Assessment and

Treatment. (5th Ed.). F.A. Davis Company, 1915 Arch Street, Philadelphia.

Ozer, M.N., Payton, O.D., Nelson, C.E.

2000 Treatment Planning for Rehabilitation: A

Patient-Centered Approach. McGraw-Hill, New York. 